\title{
The active working shoot modeling profile of the centrifugal microturbine
}

\author{
Nikolai N. Efimov ${ }^{1}$, Sergei V. Skubienko ${ }^{1}$, Vadim V. Kopitsa ${ }^{1,}{ }^{*}$, Igor Y. Kolodyazhny ${ }^{1}$, \\ Evgeny A. Anisimov ${ }^{1}$, and Ivan I. Ermakov ${ }^{2}$ \\ ${ }^{1}$ South-Russian State Polytechnic University (Novocherkassk Polytechnic Institute) M.I. Platova, \\ 346400 Novocherkassk, Russia \\ ${ }^{2}$ Kuban State University, 350040 Krasnodar, Russia
}

\begin{abstract}
For the small distributed power, microturbines of electric 30-500 kW capacity are used: gas piston engines, gas and steam turbines, each of which has certain advantages and disadvantages. In the work, the active working blade profile is simulated for a single-stage, two-stream, centripetal microturbine, in order to determine the optimum profile design satisfying the reliability conditions and economy. The basis is a humidsteam microturbine of a horizontal electric version with a capacity of $30 \mathrm{~kW}$. The initial data for software simulation were the microturbineactive stage characteristics, determined by the steam turbines calculation traditional methods.
\end{abstract}

\section{Introduction}

Small distributed power engineering becomes one of the important directions in the energy development strategy in the future. This is facilitated by a high level of the power engineering industry, active development of non-traditional energy based on the use of renewable energy sources (solar, wind, etc.), as well as the need and the need for small energy in certain conditions. For example, in the autonomous mode of operation, and as additional electricity production for own needs. In the consumption practice in recent years have been used micro-power plants of low power $30-500 \mathrm{~kW}$ : gas piston engines, gas and steam turbines. If gas-piston and gas micro-power plants of well-known companies (Capstone Turbine Corporation (USA) [1], lngersoll Rand (USA) [2]), OPRA (Holland), etc. [3]) are already applied, steam power low-power power plants the regenerative cycle of Rankin, are just beginning to be introduced. Nevertheless, steam power plants have certain advantages over gas and gas piston engines. When using cogeneration and trigeneration, they are able to create not only electricity, but heat and cold in the required quantity. In this regard, it should be noted that gas piston aggregates typically produce $1 \mathrm{~kW}$ of electric power per $\mathrm{kW}$ of heat; Gas turbines are able to increase this ratio to $2.0 \mathrm{~kW}$. When using steam power plants, this ratio increases to $10-12.0 \mathrm{~kW}[4,5,6,7]$.

\footnotetext{
* Corresponding author: vadimnpi@mail.ru
} 


\section{Problem statement}

The greatest importance in the microturbinesdesign is working blades, as the main link in the energy transformations chain, leading to the final result - the electricity production. Working blades profiling determines how economical and reliable the turbine's working processes are. In the present work, the microturbine blades profiling is simulated to optimize the working blade profile of a centripetal two-flow turbine. For the initial version of such a turbine, the nozzle and working channels profiles were determined on the basis of recommendations [8]. However, the strength calculations of such blades have shown that they experience large centrifugal loads comparable to the maximum permissible. Therefore, the task was set to develop a more reliable working blade profile of the active pressure stage for a centripetal microturbine with an electric capacity of $30-50 \mathrm{~kW}$. Technical characteristics of a single-stage, active radial steam turbine with an electric power of $30 \mathrm{~kW}$, calculated according to the procedure [9], are presented in Table 1.

Table 1. Characteristics of the turbine

\begin{tabular}{|c|c|c|}
\hline $\begin{array}{c}\text { No } \\
\mathbf{p} / \mathbf{p}\end{array}$ & Indicators & $\begin{array}{c}\text { Initial version } \\
\mathbf{5} \text { nozzles per flow }\end{array}$ \\
\hline 1 & Initial pressure $\mathrm{p}_{0}, \mathrm{MPa}$ & 1,0 \\
\hline 2 & The temperature of wet steam at the inlet. $\mathrm{t}_{0},{ }^{\circ} \mathrm{C}$ & 180,0 \\
\hline 3 & Final pressure $\mathrm{pk}, \mathrm{MPa}$ & 0,$12 ; \mathrm{t}_{\text {нас }}=104{ }^{\circ} \mathrm{C}$ \\
\hline 4 & Steam flow rate to the turbine $\mathrm{G}(2$ flows $), \mathrm{kg} / \mathrm{s}$ & $0.0946 \times 2=0.1892$ \\
\hline 5 & Diameter of the impeller at the inlet to the working blades, $\mathrm{d}_{1}, \mathrm{~m}$ & 0.300 \\
\hline 6 & Diameter at the outlet of the working blades, $\mathrm{d}_{2}, \mathrm{~m}$ & 0.250 \\
\hline 7 & Rotor speed, $\mathrm{n}, \mathrm{rp} / \mathrm{m}$ & 12000 \\
\hline 8 & Disposable heat transfer of stage $\mathrm{H} 0, \mathrm{~kJ} / \mathrm{kg}$ & 363.45 \\
\hline 9 & Absolute velocity at the exit from the nozzle array, $\mathrm{c} 1, \mathrm{~m} / \mathrm{s}$ & 784.38 \\
\hline 10 & Output height of nozzle blades, $11, \mathrm{~m}$ & 0.010 \\
\hline 11 & Output height of working blades, $12, \mathrm{~m}$ & 0.016 \\
\hline 12 & Nozzle channels number, $\mathrm{z} 1$ & $5 \times 2$ \\
\hline 13 & Working blades number, $\mathrm{z} 2$ & $52 \times 2$ \\
\hline 14 & Relative internal efficiency of the stage, $\eta$ oi, & 0.484 \\
\hline
\end{tabular}

\section{Method of solution}

By changing the model parameters, it is possible to obtain the optimum blade profile shape, which will satisfy the gas dynamic and strength requirements. One of the main issues in this case is the optimal approach choice to constructing a geometric profile model (topology). The results of this approach can be used to develop profiling principles based on the inverse problem solution.

When designing the impeller, the initial data were determined: the blade height, the meridional contour length of the blade, the angle of the flow entrance, the distance from the nozzle, etc. These limitations were dictated primarily by the geometric characteristics of the entire microturbine complex (distance from the upper edge of the blade to the cover, the 
impeller width, the distance from the center of the shaft to the nozzle grid, etc.). The resulting sketch of the impeller with blades is shown in Fig. 1.

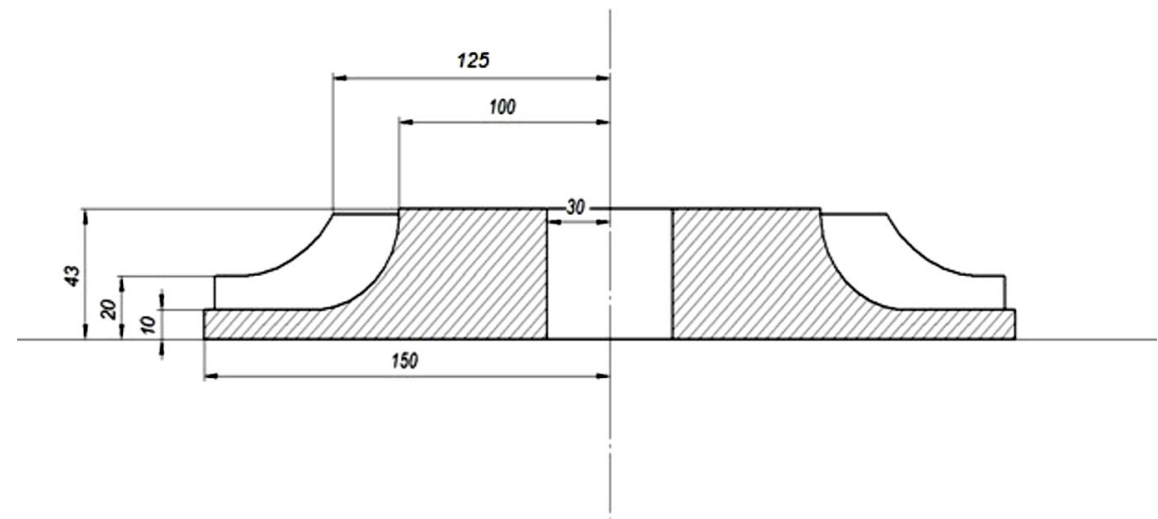

Fig. 1. Design of impeller with blades.

The impeller adopted for modeling has 26 working blades located throughout the hub circumference with the same pitch between them. The geometry of the nozzle apparatus did not undergo any initial changes, and was taken in the original version as given. The nozzle apparatus itself is represented by five Laval nozzles arranged around the impeller. A nozzle grate sketch with channels is shown in Fig. 2.
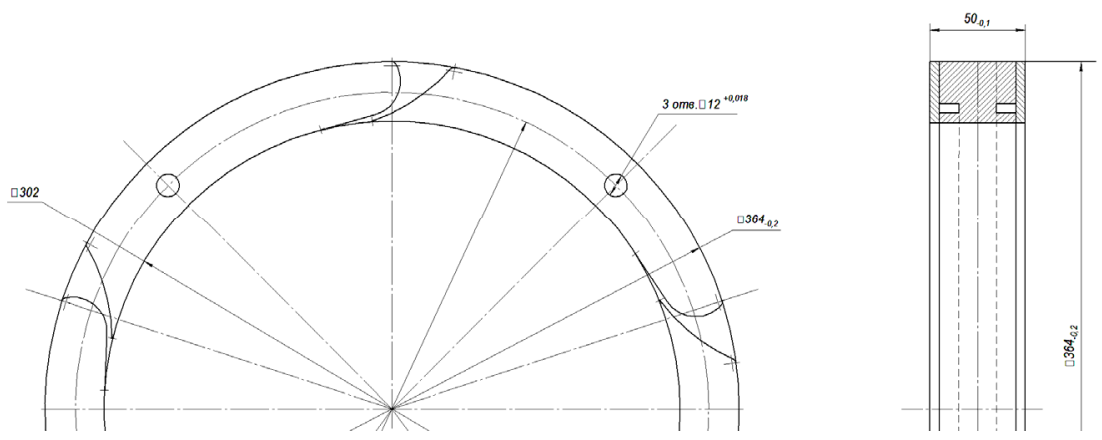

Fig. 2. Nozzle array with channels.

The presented sketches were used as the starting boundaries for extrusion operations and the construction of sections on surfaces.

The impeller of such a turbomachine is located in the ZX plane, and the rotation is carried out around the $\mathrm{Z}$ axis. The wet steam flow input occurs vertically along the $\mathrm{X}$ axis to the center of the global coordinate system (see Fig. 2). The geometric model of the blade was constructed using four main impeller points (Table 2, Fig. 1), corresponding to the places where the profile was applied to the suction loop and the peripheral one, at the inlet and outlet of the flow.

Table 2. Basic settings for profile settings

\begin{tabular}{|c|c|}
\hline Type & RadialTurbine \\
\hline P1 - HubInlet, Z, R, mm & 10,150 \\
\hline P2 - ShroudInlet, Z, R, mm & 20,150 \\
\hline P3 - HubOutlet, Z, R, mm & 50,100 \\
\hline P4 - Shroud Outlet, Z, R, & 50,125 \\
\hline
\end{tabular}


The geometrical model of a turbomachine with nozzles and a flowing part is shown in Fig. 3.

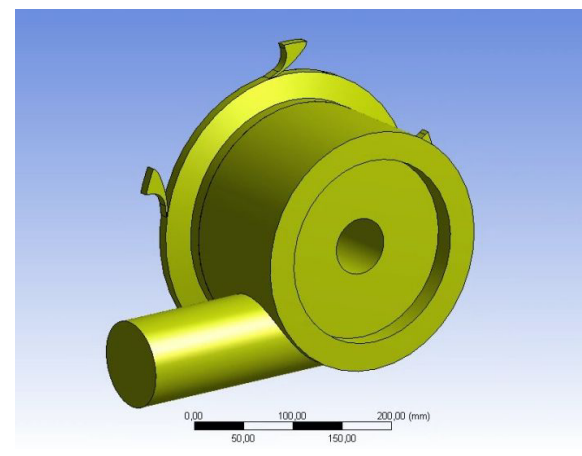

a)

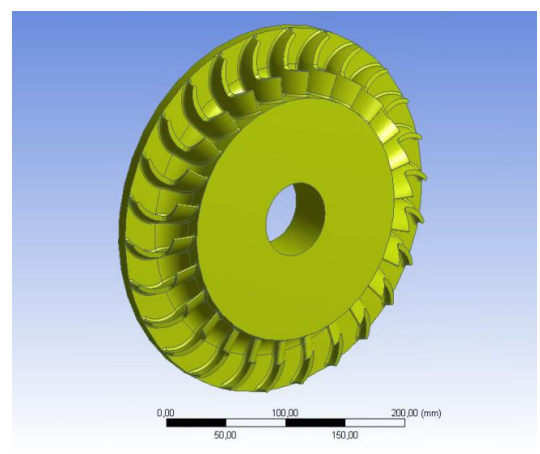

b)

Fig. 3. Geometric model of a turbomachine (a) and impeller with blades (b).

For some media, viscous stresses can be expressed as a function of the local or total strain rate. In three-dimensional flows, the local strain rate consists of a linear and volume strain rate. All gases and many liquids are isotropic by default and the mathematical model of the wet vapor motion in a microturbine is formed on the same concept basis (the liquid is isotropic).

The SST object is a turbulence model with a vortex viscosity in two equations, which is suitable for flows with unfavorable pressure gradients. When the flow enters the blade region, the pressure decreases as the velocity increases when the flow enters the blade feather cavity. The pressure reaches a local minimum on the blade crest and begins to increase again, which leads to an unfavorable pressure gradient for the flow, that is, $\mathrm{dp} / \mathrm{dx}>0$.

In such flows, the standard model k- $\varepsilon$ overrides the level of turbulent shear stress due to too long a scale length. The $\mathrm{k}-\omega$ model does not require any damping functions, whereas $\mathrm{k}-$ $\varepsilon$ requires complex non-linear damping functions for the wall layers.

Since the two models have different strengths and weaknesses, a hybrid model was developed to combine the most accurate modes of the two models, which led to the development of a two-model viscosity system SST k- $\omega$. In the k- $\omega$ system, the process model is used in the solid wall surface vicinity and is transformed into k- $\varepsilon$ far from it. The ability to combine the strengths of k- $\omega$ and $k-\varepsilon$, the SST k- $\omega$ system becomes an appropriate choice for unfavorable pressure gradients.

The grid model obtained in the TurboGrid is transported to the CFX proprietor, where it is prepared for calculation. In this case, the boundary conditions are specified, the working body and the domain are adjusted. Parameters are specified at the input and output to the inter-blade region, the impeller rotational speed, the working fluid characteristics, and the solver parameters.

Since the blade profile was created using special BaldeModeler options, a special Turbo Mode module will be used to configure the task, where convenient setting of all model operating conditions is available.

According to the received settings, the preprocessor assigns the necessary interactions of the boundary areas, as well as the domain basic settings. The working body Wet Steam was created independently by the substance chosen model from the list of working bodies proposed types.

The results are shown in the velocity form and pressure fields in the two sections, as well as the current lines velocity on the entire domain. The velocity fields in the perpendicular and longitudinal cross sections shown in Fig. 4. The pressure field are shown in Fig. 5. The speeds in domain Streamlines are shown in Fig. 6. 


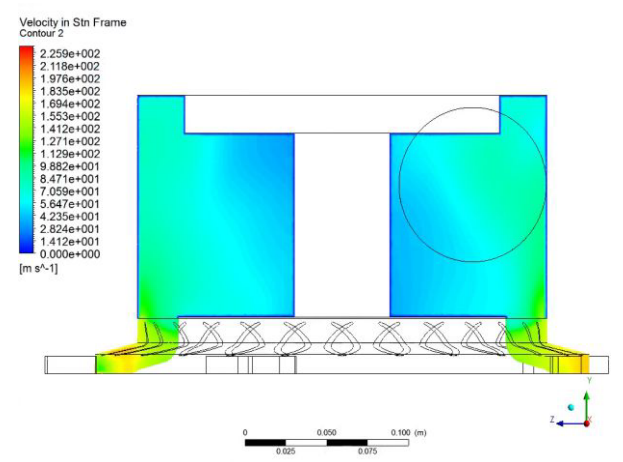

a)

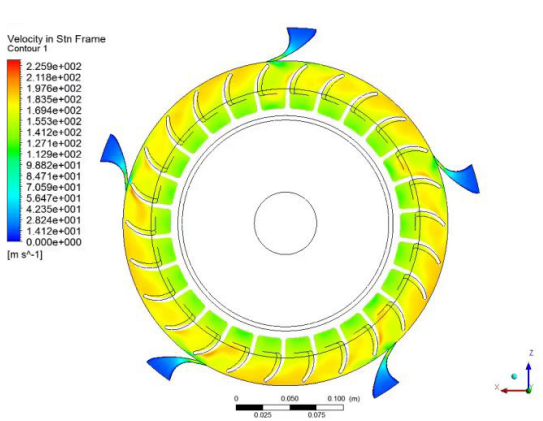

b)

Fig. 4. The velocity field in the longitudinal (a) and transverse (b) sections.

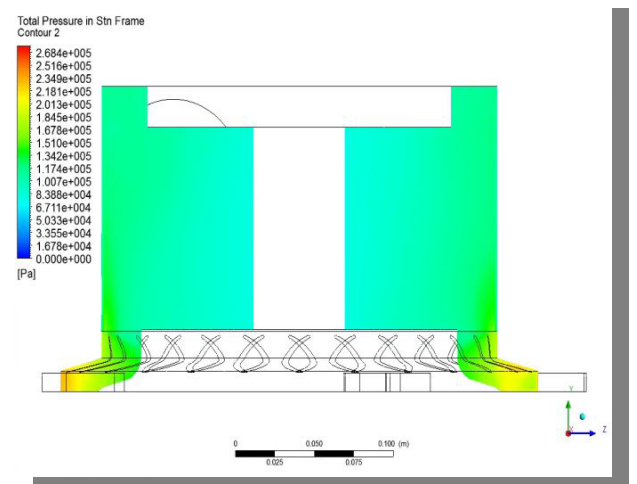

a)

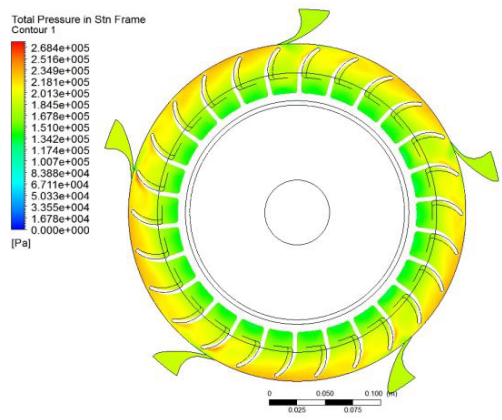

b)

Fig. 5. Field pressures in longitudinal and cross sections.

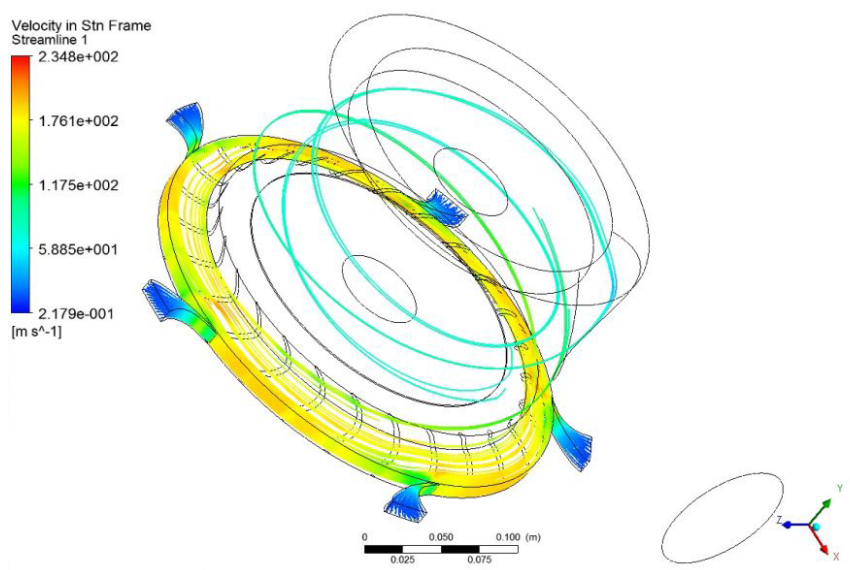

Fig. 6. Velocities Streamlines domain. 


\section{Results}

The simulation flow part the microturbine has been determined optimum blade profile, allowing smoothly redirect the flow of the wet steam flow in the active part the microturbine pressure stage of radial centripetal trajectory in the axial direction with minimal output speed loss. Thus such a construction work turbine blades will have higher strength properties.

\section{References}

1. https://www.capstoneturbine.com.

2. http://www.irena.org/DocumentDownloads/Publications/RE_Technologies Cost_Analysis-BIOMASS.pdf.

3. http://www.opraturbines.com/en/About-OPRA-Turbines.

4. O. R. Kuznetsova, Economic efficiency of decentralized power supply systems: a case Krai. Disc. cand. econ. (Komsomolsk-on-Amur, 2002)

5. N. N. Efimov, Power. Specialized magazine, 6, 54 (2013)

6. N. N. Efimov, V.I. Parshukov, V. V. Papin, R. V. Bezuglov, I. V. Yanchenko, R. A. klinnikov, D. Yu. Chumakov, E. S. Trofimchenko, Math. universities of North-Kavko. region. Techn. Science, 1, 51 (2013)

7. N. N. Efimov, V.I. Parshukov et al., IJPAM, 22, 149 (2017)

8. M. E. Daich, G. Filipov, Axial turbines atlas profiles lattices (Mechanical Engineering, Moscow, 1965)

9. A. Kostiuk, V.V. Frolov, A.E. Bulkin, A. D. Truhny, Steam and gas turbines for power plants: the textbook for high schools, 3rd ed. (Publishing House MEI, Moscow, 2008) 